$63^{\text {ème }}$ Congrès de la SFCO, 03028 (2015)

DOI:10.1051/sfco/20156303028

(C) Owned by the authors, published by EDP Sciences, 2015

\title{
POSTER
}

\section{Un adénome pléomorphe labial}

\author{
Rochefort J*, Agbo-Godeau S**, Goudot P**, Descroix V* \\ * Unité de Chirurgie Buccale, service Odontologie de l'hôpital Pitié Salpêtrière \\ * Département de Pathologie de la Muqueuse Buccale, service de Stomatologie et Chirurgie Maxillo-Faciale, \\ Hôpital Pitié Salpêtrière
}

L'adénome pléomorphe est une tumeur bénigne des glandes salivaires, à double composante, épithéliale et mésenchymateuse. Il représente 45 à 65 \% des tumeurs bénignes des glandes salivaires. Il se développe le plus souvent au niveau des glandes salivaires principales, surtout la parotide, mais il peut également atteindre les glandes salivaires accessoires, comme celles du palais ( $5 \%$ des cas), ou plus rarement de la lèvre supérieure. Sa prévalence est plus importante chez les hommes et il touche les jeunes adultes avec une moyenne d'âge de 37 ans (El Khatib 2011). Il est souvent asymptomatique, à croissance lente et intermittente. Le nodule est ferme sans être dur, non fixé et bien circonscrit dans la plupart des cas. Les nodules labiaux d'allure bénigne sont le plus souvent retirés par simple exérèse chirurgicale et l'analyse histologique en précise la nature. La récidive fréquente des adénomes pléomorphes, fait souvent suite à une exérèse incomplète (Reychler 1991, Dechaume 1967) d'où l'importance d'une prise en charge thérapeutique rigoureuse.

Le cas décrit concerne un homme de 37 ans, informaticien, adressé pour une gêne au niveau de la lèvre supérieure droite persistante depuis 4 mois. Il a présenté un spina bifida congénital au niveau de L4-L5 ayant nécessité de multiples opérations et entraîné une paralysie des membres inférieurs. Il n’a pas d'intoxication alcoolo-tabagique ni d'allergie. Aucune médication n'est en cours. A l'examen clinique exo-buccal, on note une légère voussure de la lèvre supérieure droite, correspondant à un nodule au niveau de la face interne muqueuse. Ce nodule est ferme, asymptomatique et mesure $1 \mathrm{~cm}$ de plus grand diamètre. Il est bien délimité, et non fixé, ni sur les plans profonds ni sur la muqueuse labiale, dont l'aspect est semblable à celui de la muqueuse saine.

Il est décidé de procéder à l'exérèse de la lésion. Sous anesthésie locale, l'intervention consiste en une simple incision superficielle de la muqueuse de $1.5 \mathrm{~cm}$ de long, permettant d'extérioriser le nodule par pression puis de le disséquer facilement des glandes salivaires accessoires avoisinantes. Deux points de suture sont réalisés.

L'analyse histologique précise qu'il s'agit d'une lésion nodulaire bien limitée, correspondant à une prolifération mixte épithéliale et mésenchymateuse avec une fine capsule qui ne présente pas d'effraction. L'examen conclut à la confirmation histologique d'un adénome pléomorphe, encapsulé dont l'exérèse est complète.

A ce jour, 3 ans après, aucune récidive n’a été signalée chez ce patient.

This is an Open Access article distributed under the terms of the Creative Commons Attribution License 4.0, which permits unrestricted use, distribution, and reproduction in any medium, provided the original work is properly cited. 
L'exérèse de cet adénome pléomorphe a pu être réalisée du fait de sa petite taille, de sa facilité d'accès et d'extériorisation. Lors de l'abord chirurgical, si les limites capsulaires avaient été moins nettes et la dissection moins aisée, une biopsie aurait été préférée afin de préciser la nature histologique de la lésion ; l'intervention chirurgicale, dans un second temps, permettant d'effectuer une exérèse impliquant des marges à distance du nodule, afin d'éviter toute effraction capsulaire ou dissémination glandulaire, principaux facteurs de risque de récidive dans le cas de ces tumeurs salivaires bénignes.

Nom et adresse du conférencier

\section{Juliette ROCHEFORT}

Unité de Chirurgie Buccale, service Odontologie de l’hôpital Pitié Salpêtrière

47-83 Bd. de l'hôpital

75013 Paris (France)

julietterochefort@yahoo.fr 